\title{
CK syndrome
}

INSERM

\section{Source}

INSERM. (1999). Orphanet: an online rare disease and orphan drug data base. CK syndrome. ORPHA:251383

CK syndrome is a rare, genetic, X-linked syndromic intellectual disability disorder characterized by mild to severe intellectual disability, infancy-onset seizures, post-natal microcephaly, cerebral cortical malformations, dysmorphic facial features (including long, narrow face, almond-shaped palpebral fissures, epicanthic folds, high nasal bridge, malar flattening, posteriorly rotated ears, high arched palate, crowded teeth, micrognathia) and thin body habitus. Long and slim fingers/toes, strabismus, hypotonia, spasticity, optic disc atrophy, and behavioral problems (aggression, attention deficit hyperactivity disorder and irritability) are additional features. 